\title{
Periorbital Rejuvenation: The Egyptian Experience in the Management of Dark Halos
}

\author{
ALY HUSSEIN S. ABULHASSAN, M.B.B.Ch.; HAMED M. KADRY, M.D.* and \\ HUSSEIN S. ABULHASSAN, M.D.** \\ The Department of Plastic \& Reconstructive Surgery, Faculties of Medicine, Cairo* and Alexandria** Universities
}

\begin{abstract}
Background: The periorbital region represents a challenging area for the plastic surgeon since ages. The complain of the dark halos of our patient received a continuous attention with trials to modify the discolouration.

Aim of Study: The new technique of nanofat grafting of the region by SNIF technique is a new way of managing the area properly. The nanofat with its content of fine fat and stem cells will help improve the color and quality of the eyelid region.
\end{abstract}

Material and Methods: In this study 64 Egyptian male and female patients of various age groups were involved in our study with satisfactory results in most cases.

Follow-up: All cases were evaluated from 6m-1 year.

Recommendation: This technique is recommended for the management of dark halos.

Key Words: Periorbital - Rejuvenation - Egyptian - Dark halos.

\section{INTRODUCTION}

Periorbital dark halos are an increasing cosmetic problem with many individuals looking for a medical solution of this disturbing problem.

They were defined as infra-orbital dark circles (halos) that present with relative darkness of the eyelids. The anatomy and the aging changes of this area is a critical component to successful rejuvenation [1].

The periorbital region is one of the most dynamic and unforgiving areas in relation to volume enhancements. The eyelid skin is the thinnest in the body that lacks subcutaneous fat and is a dynamic structure. Irregularities are more readily apparent in this area than other areas of the face. Understanding the complex periorbital anatomy is important to help avoid potential complications
[2,3]. As this condition affects individuals of varying age groups, both sexes and all races, it worsens with aging process $[4,5]$. The dark circles can influence the quality of life of persons since it gives the impression of looking tired, sad or hung over drugs [13].

Possible causative factors include excessive pigmentation; thin, translucent eyelid skin overlying the orbicularis oculi muscle; and shadowing due to skin laxity and tear trough. Excessive pigmentation is associated with dermal melanocytosis, post inflammatory hyperpigmentation 2ry to atopic or allergic contact dermatitis. Other causes are familial or the excessive subcutaneous hypervascularity theory.

Treatment modalities showed problems that annoy both the patient and the physician, it included a variety of alternatives that vary in effect and duration but can not guarantee patient satisfaction.

The list includes a variety of treatment options including:

- Topical Agents: Hydroquinone, topical retinoic acid $0.01 \%$ to $1 \%$ and steroids.

- Chemical Peeling: Glycolic acid with variable concentrations.

- Lasers: All types with recent good effect of fractional $\mathrm{CO} 2$ effect.

- Surgery: Including blepharoplasty of the lower and upper excess dark skin in older age groups but fail to help the younger patients.

- Periorbital Injectables: Both botulinum toxin and dermal fillers have been widely used to address periorbital aging. Complications arising from botulinum toxin injections are usually transient and related to its effect on the affected muscles. Most patients with complications arising 
from botulinum toxin injections can be treated supportively with ocular drops and referral to an ophthalmologist or oculoplastic surgeon may be considered [6].

In recent years a new and effective way of harvesting a very minute particles of fat cells and adipose derived stem cells which was named $\mathrm{Na}-$ nofat graft or supermicrofat graft were introduced by the work of Tonnard et al., 2014 [7].

To ensure an easy and successful method of injection, the harvested fat has to be very fine with very small fine fat particles.

The initial goal of fat grafting was to treat volume losses created by disease, trauma, or aging. Fat was injected with relatively large blunt cannulas (A) $2 \mathrm{~mm}$ diameter). For delicate areas such as eyelids and lips, smaller injection cannulas became popular. Lipofilling with cannulas as small as $0.7 \mathrm{~mm}$ in diameter, also called microfat grafting, has been described [8-11].

We previously described microfat grafting in the deep dermal layer of the skin with 23-gauge sharp needles for treatment of fine rhytides in the face [12].

A multiperforated liposuction cannula $3 \mathrm{~mm}$ in diameter with 2-mm side holes was used to harvest fat for injection of other parts of the face [9].

Nguyen et al., described the use of a similar multiperforated harvesting cannula with side holes of $1 \mathrm{~mm}$ [10]. Coleman and Mazzola reported the use of injection cannulas up to 22 gauge [9].

Nguyen et al., applied blunt injection 25-gauge cannulas in a mouse model and mentioned the use of blunt 21- or 23-gauge cannulas for fat grafting in clinical cases [10].

The initial goal of local injections with nanofat using 27-gauge needles was to use it as a new modality of treating dark halos under the eye.

Aim of the work to study the effect of Nanofat (supermicro fat) grafting to the lower dark pigmented lower eyelids resistant to medical treatment.

\section{MATERIAL AND METHODS}

This study included 64 patients of adult males and females with dark pigmented circles around their eyes in the period 2013-2015.

Our patients were selected from the outpatient clinics of both Qasr Elini Hospitals Plastic Surgery Clinics and Alexandria Main University Hospitals
Plastic Surgery Clinics from February 2013-2015. Each case was followed-up for 6 months or more regarding the improvement in skin discoloration, skin dryness, wrinkles and local irritation manifestations.

These patients (64) were 50 females and 14 males with age ranged between 18-36 years old. Each patient was subjected to a complete medical examination and surgical evaluation, thorough explanation of the procedure, consent of the patient

The procedure was done in the operating room with local or general anesthesia, prepping and drapping of both the abdomen or knees or back for the fat harvest and the face as the site of injection.

Eye shield or eye ointment or gel was used frequently to protect the eyes during the procedure.

Finally, a very detailed counseling and preoperative and postoperative photographic documentation as well as psychological evaluation of the patients were done.

\section{Fat harvest:}

Surgical technique:

Using a number 11 blade a 2-mm stab incision was made along the skin-tension lines where fat was harvested after infiltration with a tumescent solution (1 liter of normal saline sodium chloride with 1:1,000,000 adrenaline). Liposuction was performed either manually and collected in sterile syringes or using high negative pressure vacuum with a suction device and a sterile canister.

Sites of fat harvesting varied from lower abdomen (infraumbilical), love handles, inner thighs and thoracic back.

It was reported that the thoracic back has the largest number of adipose derived stem cells as discovered by Kishi et al., [13].

Volumes of fat was less than $10 \mathrm{~mL}$, the fat was harvested with a cannula on a $10 \mathrm{~mL}$ Luer-Lok syringe. Volumes of more than $10 \mathrm{~mL}$ were harvested with a suction device and a sterile canister.

The suction cannula had a diameter of 2 or $3 \mathrm{~mm}$, with multiple sharp 1-mm 15-20 holes at its top segment.

The limited diameter of the cannula holes is important for obtaining small particles to be injected through a 23-gauge needle [12]. 


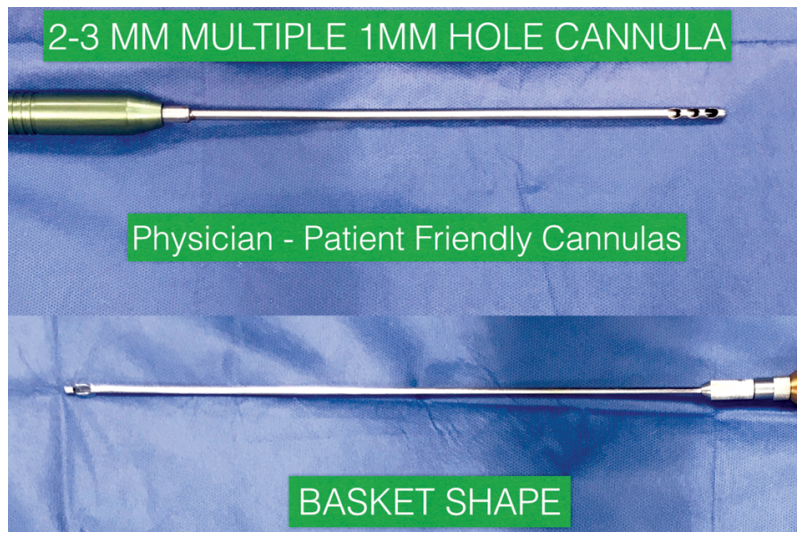

Fig. (1): The harvesting cannula consists of a multiport sharp cannula, $2-3 \mathrm{~mm}$ in diameter, with holes of $1 \mathrm{~mm}$ in diameter. Or basket shaped cannula which gives a good yield of fat Note the sharp edges of the holes, which transform the cannula into a rasp, augmenting the harvesting yield of this small-holed cannula.

Fat was mounted over a sterile nylon or mesh cloth over a sterile canister and the supernatant was poured over it.

The fat was left to decant and stirred properly with a sterile spoon and then transferred into $10 \mathrm{cc}$ syringes.

The lipoaspirate was mechanically emulsified after rinsing. Emulsification of the fat was achieved by shifting the fat between two 10cc syringes connected to each other by a female-to-female Luer-Lok connector. A total of 30 times between two Luer-lock 2.5-ml syringes joined to each other by a connector with three small holes (Transfer Emulsifier; Tulip Medical, San Diego, Calif).

This was done to remove the connective tissue remnants that would block the fine needles. This effluent is called "Nanofat".

With the help of a double Luer-Lok adapter, the fat was brought into $1 \mathrm{cc}$ syringes. An $0.5 \mathrm{~mm}-0.7 \mathrm{~mm}$ blunt cannula was adjusted over the 1cc syringe. A 23 -gauge needle was mounted on the syringe for intradermal injection. plane.

The injection was made in a superficial dermal

Immediately after fat injection, the skin is blanched over the injected wrinkle. A slight overcorrection is advised because the blanching and overcorrection will normalize within a few hours, after resorption of the interstitial fluid.

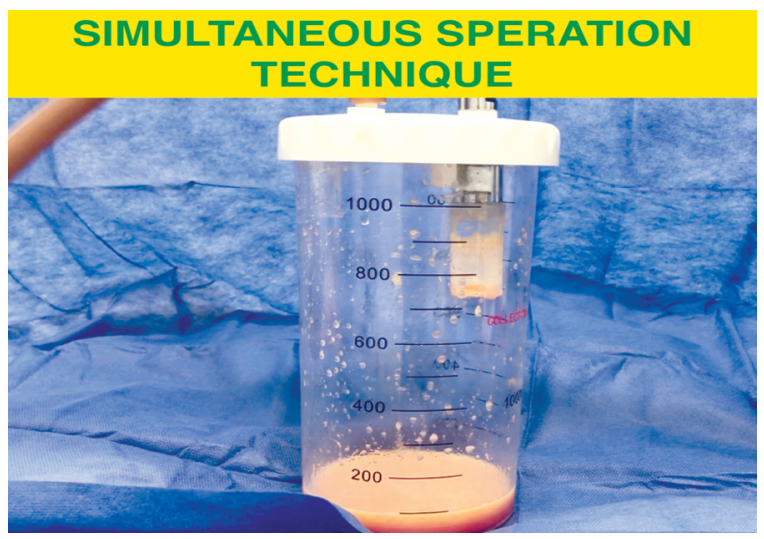

Fig. (2): A sterile canister is used for fat collection as a simultaneous seperation technique under low pressure.
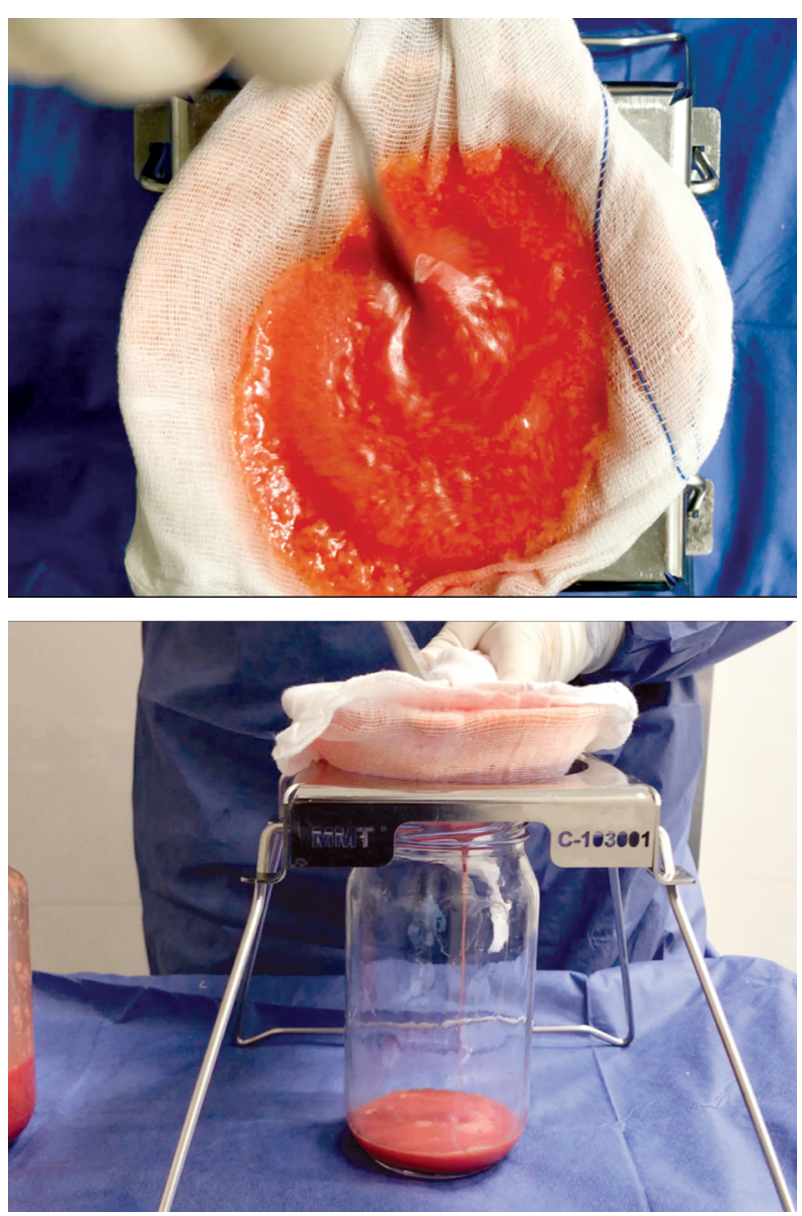

Fig. (3): Fat is stirred with a sterile spoon over a sterile mesh cloth to wash out the fat.

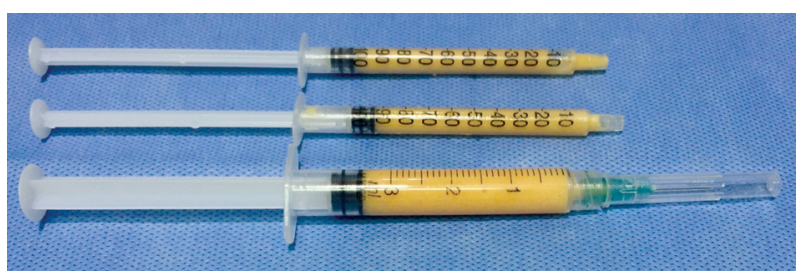

Fig. (4): Fat is then transferred to $1 \mathrm{cc}$ or $3 \mathrm{cc}$ sterile syringes using a female to female leur lock connector. 
Cold compresses were applied at the end of the injection along with antibiotic creams and should continue postoperative for 48 hours. Patients were seen every 2 days in the first week then weekly for 4-6 weeks and every month for 6 months to one year.

\section{RESULTS}

The 64 patients were 50 females $(78.13 \%)$ and 14 males $(21.8 \%)$ with age range between 22-53.

Marital status of the group was 50 unmarried and 14 married.

The main complain was looking tired and sleepless, second was inability to hide the darkness by any sort of make-up and third group was linked to drug addiction accusation by parents, teachers or friends.

54 were satisfied with a single injection of nanofat, while 10 patients requested a re-injection which was usually performed under local anesthesia upon patient request and was done after 6-8 months.

The improvement in the eyelids was monitored by the surgeon and patient and was given a score from 1-5 where 1 is poor and 5 excellent as demonstrated in table...).

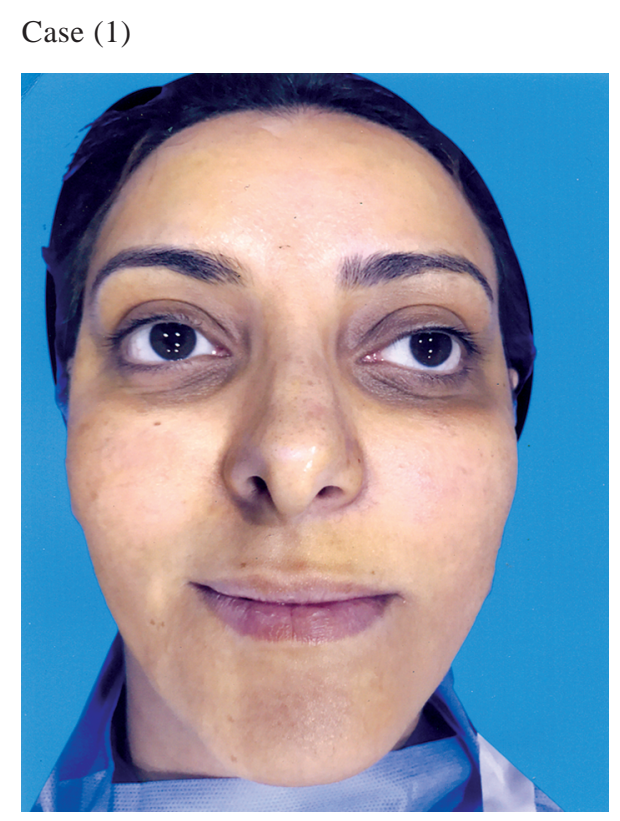

Pre operative

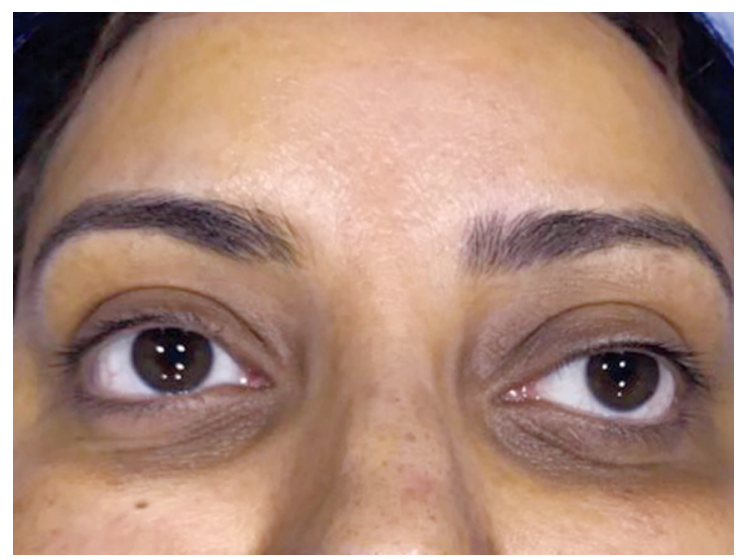

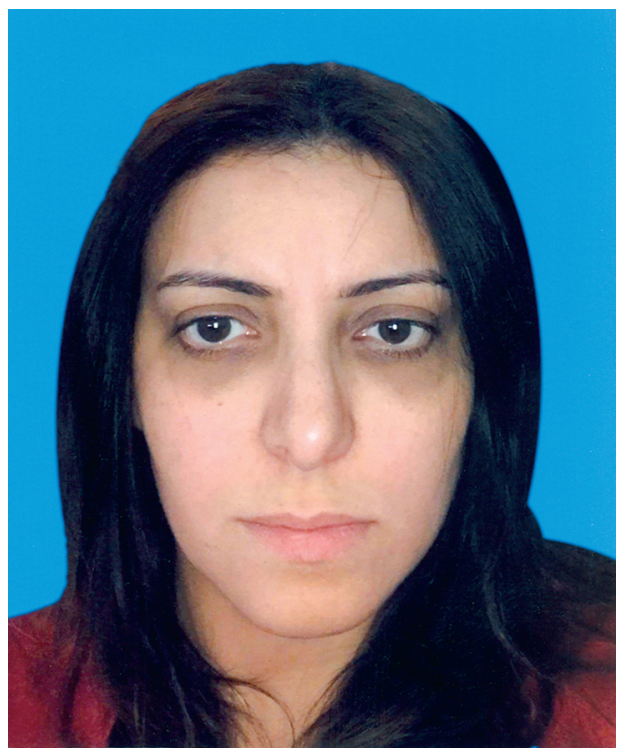

6 months post operative

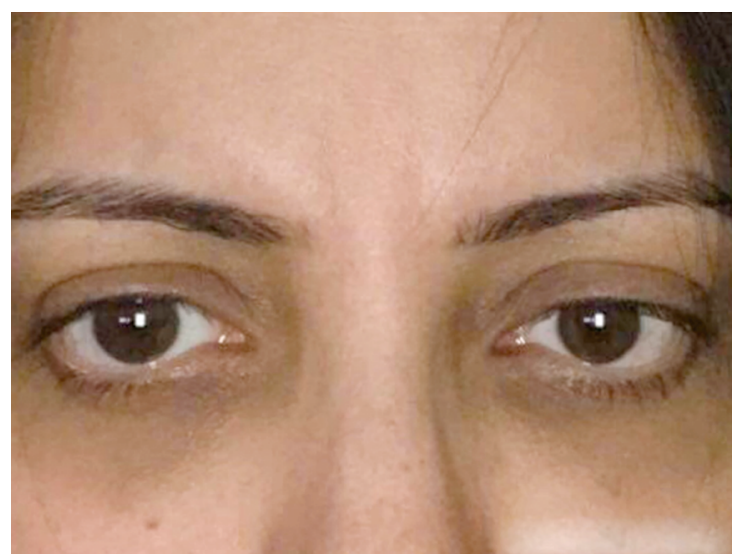

1 year post operative 
Case (2)

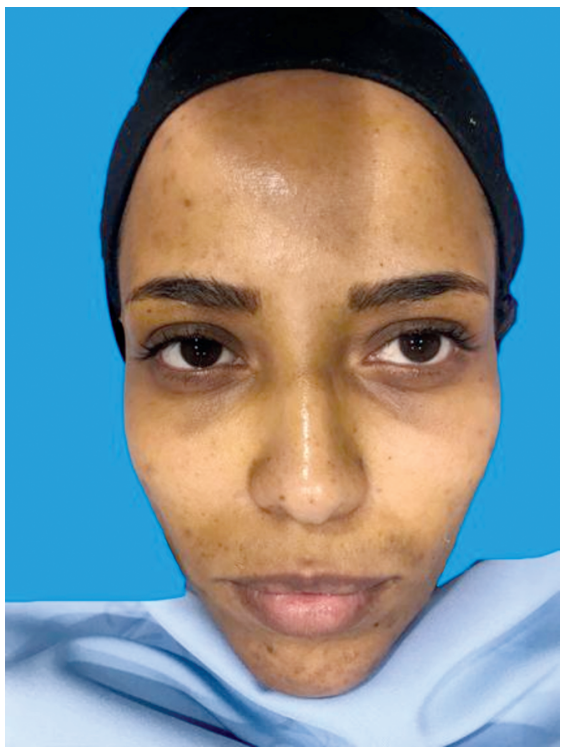

Pre operative

Case (3)

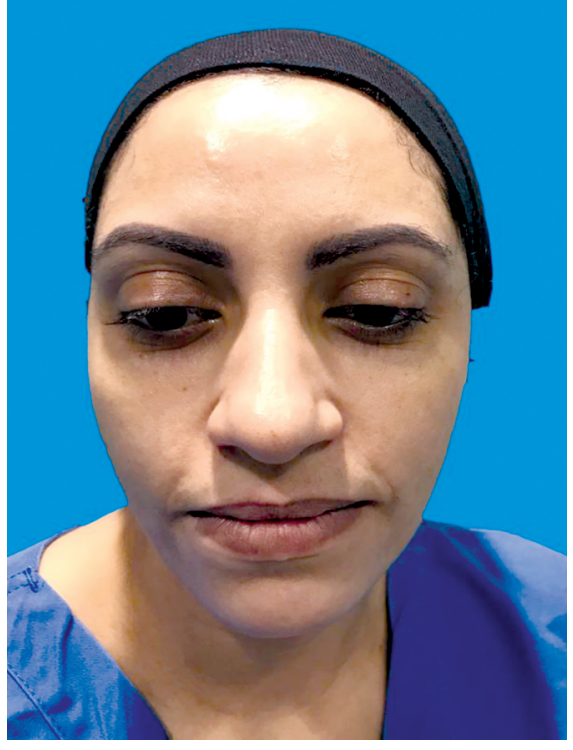

Pre operative

\section{DISCUSSION}

Periorbital dark halos are a very popular complain in the middle east and north African region variable with different skin types.

Different treatment options have been tried with a high failure rate and dissatisfaction rate.

The periorbital region of the face is an important anatomical area for any surgical and non-surgical rejuvenation procedures which includes different subunits in which the eyes are in the center Invo-

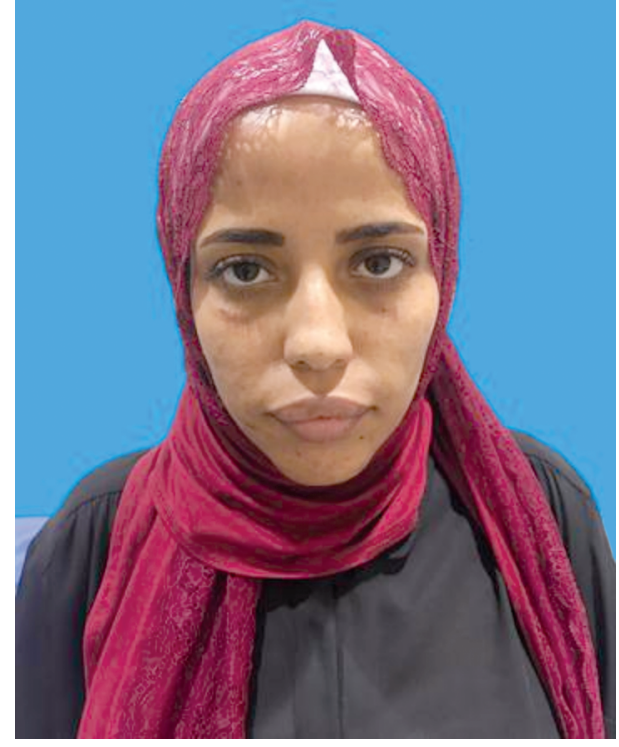

6 months post operative

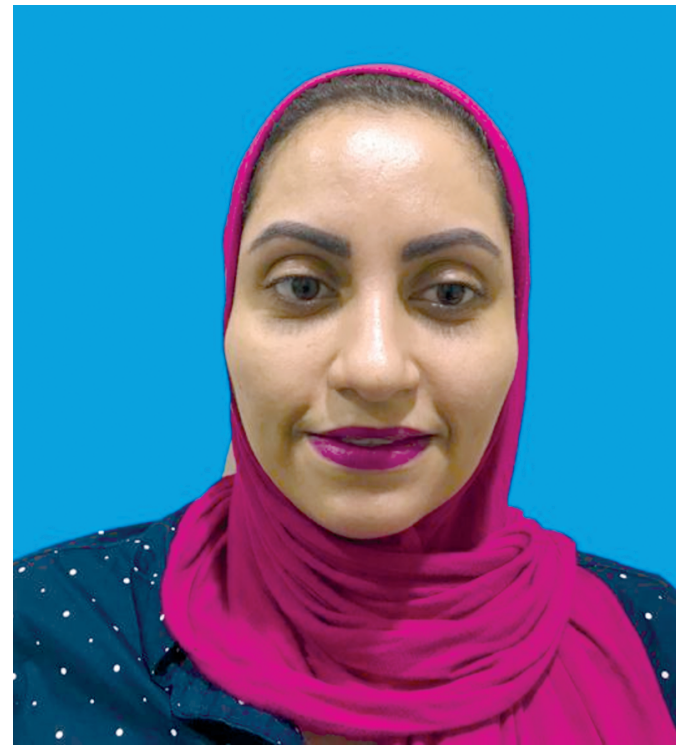

Post operative

lutional changes of eyebrow and eyelid are divided into static and dynamic components. The static component is defined as reducing the global loss of volume due to changes in bone and fat pad that support the eyebrow [14-18].

In this study we tried the surgical mini-invasive technique of nanofat or supermicrofat grafting in the lower eyelids in the subdermal layer. The use of suction cannulas with very small diameter of 2 or $3 \mathrm{~mm}$, with multiple sharp 1-mm holes at its top segment was aid for an easy injection technique. 
To provide a smooth injection through these fine cannulas, the fat particles need to be sufficiently small. If the fat particles are too large, passage through the injection cannula would be difficult [12].

To ensure a smooth fat injection through 27gauge sharp needles, the aspirated fat has to be processed mechanically to provide a liquid fat emulsion, known as nanofat. A yield of $1 \mathrm{ml}$ of nano-fat per $10 \mathrm{ml}$ of lipoaspirate can be expected using a nanofat processing procedure [12] and sharp needle fat grafting technique (SNIF) as demonstrated by (Zeltzer et al., 2012) [7].

Nanofat does not have the capacity to build up a significant fat volume. It's not a method of augmentation but of rejuvenation [12].

Consequently, nanofat is evidently not suitable for any volume replacement indications. In fact, because of the lack of adipocytes, the volumetric effect of nanofat is obviously very limited. Therefore, the indications for nanofat injection are different when compared with microfat grafting $[7,12]$.

Although both adipocytes and adipose-derived stromal cells in the graft are critical components for tissue volumization, $[\mathbf{1 9 , 2 0}]$ therapies for improving the quality of tissue (i.e., vascularity, inflammation, fibrosis, and healing capacity) may not require adipocytes [21].

Usually, nanofat grafting is combined with other modalities of microfat grafting such as sharp needle intradermal fat grafting or PRP to obtain a soft-tissue filling effect, where the nanofat is layered more fanwise in an intradermal level to enhance the skin quality. In our experience, as opposed to microfat, the effect of nanofat usually appears with a delay of 4 weeks to 3 months.

In the case where dark circles were treated, the beneficiary effect was preceded by a rather prolonged erythematous phase, which most likely is attributable to the soft-tissue rearrangement.

Other studies used lipofilling as an instrument for skin regenerative purposes such as treatment of radiotherapy ulcers or scars [22-24]. One study reported a clear and statistically significant improvement in dermal elasticity after injection of facial scars in 14 patients [23]. The mechanism for this regenerative effect on damaged skin remains unknown. Improved elasticity is presumably a consequence of increased collagen and elastin synthesis and remodeling. These effects are most likely triggered by stem cells rather than by grafted adipocytes. Moreover, the nanofat sample analysis revealed that adipocytes were destroyed during the emulsification process.

In fact, it may be questioned whether a nanofat transfer actually is a "fat grafting" procedure, as adipocytes did not survive the emulsification process. The major effect of nanofat injection is probably a stem cell activity. Likewise, nanofat injection might rather be considered as an in vivo tissueengineering process. It might be logical to discard the dead adipocyte fraction from the nanofat and to inject the purified stromal vascular fraction only. However, isolating the stromal vascular fraction out of the nanofat before injection in routine clinical cases would be time consuming, complicated, and expensive [12].

Besides, it requires specific laboratory equipment and experience. Moreover, it is known that apoptotic cells release cytokines and attract macrophages that induce growth factors and play an important role in regeneration of the damaged tissue. Thus, co-injection of fragmented adipocytes might have a stimulating effect on stem cell differentiation and tissue regeneration [25].

If the magnitude of adipocyte reduction is small, the product might be good for tissue volumization, as during adipose-derived stromal cellenriched fat grafting [26,27]. If adipocyte reduction is greater, the micronized product might be a useful injectable tool for tissue revitalization/fertilization or immunomodulation [28].

Moreover, it is known that apoptotic cells release cytokines and attract macrophages that induce growth factors and play an important role in regeneration of the damaged tissue [25]. Thus, co-injection of fragmented adipocytes might have a stimulating effect on stem cell differentiation and tissue regeneration $[\mathbf{1 2 , 2 9 ]}$.

Most patients required one setting (84.5\%). While a second or a third injection setting was performed for the rest. The patient satisfaction in this study showed that 10 patients gave a rate of $5(15 \%)$ while 27 patients gave a rate of $4(43.9 \%)$

The procedure was performed as described by Tonnard et al in the first few cases but after various reports of filler and fat emboli in the retinal arteries causing blindness we shifted to our technique of sharp entry point and blunt cannula ramification with very minimal pressure in fat delivery in the eyelids [29]. 
In conclusion: We feel that our technique of sharp entry point and blunt cannula ramification with very minimal pressure in nanofat delivery in the eyelids improves the results of rejuvenation and treatment of dark halos in the eyelids and obtained a satisfying results in a good number of patients.

\section{REFERENCES}

1- Bravo B.S., Rocha C.R., Bastos J.T. and Silva P.M.: Comprehensive treatment of periorbital region with hyaluronic acid. J. Clin. Aesthet. Dermatol., 8 (6): 30-5, 2015.

2- Funt D. and Pavicic T.: Dermal fillers in aesthetics: An overview of adverse events and treatment approaches. Plast. Surg. Nurs., 35 (1): 13-2, 2015.

3- Cohen J.L.: Understanding, avoiding, and managing dermal filler complications. Dermatol. Surg., 34 (Suppl 1): S92-9, 2008.

4- Karacalar A., Korkmaz A., Kale A. and Kopuz C.: Compensatory brow asymmetry: Anatomic study and clinical experience. Aestheti.c Plast. Surg., 29 (2): 119-23, 2005.

5- Knize D.M.: An anatomically based study of the mechanism of eyebrow ptosis. Plast. Reconstr. Surg., 97: 132133, 1996.

6- Kashkouli M.B., Abdolalizadeh P., Abolfathzadeh N., Sianati H., Sharepour M. and Hadi Y.: Periorbital facial rejuvenation; applied anatomy and pre-operative assessment. J. Curr. Ophthalmol., 29 (3): 154-68, 2017.

7- Zeltzer A.A., Tonnard P.L. and Verpaele A.M.: Sharpneedle intradermal fat grafting (SNIF). Aesthet. Surg. J. 32 (5): 554-61, 2012.

8- Trepsat F.: Midface reshaping with micro-fat grafting. Ann. Chir. Plast. Esthet., 54 (5): 435-43, 2009.

9- Coleman S.R. and Mazzola R.F.: Fat Injection: From Filling to Regeneration. St. Louis: Quality Medical, 2009.

10- Nguyen P.S., Desouches C., Gay A.M., Hautier A. and Magalon G.: Development of micro-injection as an innovative autologous fat graft technique: The use of adipose tissue as dermal filler. J. Plast. Reconstr. Aesthet. Surg., 65 (12): 1692-9, 2012.

11- Dasiou-Plakida D.: Fat injections for facial rejuvenation: 17 years experience in 1720 patients. J. Cosmet. Dermatol., 2 (3-4): 119-25, 2003.

12- Tonnard P., Verpaele A., Peeters G., Hamdi M., Cornelissen M. and Declercq H.: Nanofat grafting: Basic research and clinical applications. Plast. Reconstr. Surg., 132 (4): 1017 26, 2013.

13- Kishi K., Imanishi N., Ohara H., Ninomiya R., Okabe K., Hattori N., et al.: Distribution of adipose-derived stem cells in adipose tissues from human cadavers. J. Plast. Reconstr. Aesthet. Surg., 63 (10): 1717-22, 2010.

14- Lemke B.N. and Stasior O.G.: The anatomy of eyebrow ptosis. Arch. Ophthalmol., 100 (6): 981-6, 1982.

15- Angelos P.C., Stallworth C.L. and Wang T.D.: Forehead lifting: State of the art. Facial. Plast. Surg., 27 (1): 50-7, 2011.
16- Presti P., Yalamanchili H. and Honrado C.P.: Rejuvenation of the aging upper third of the face. Facial. Plast. Surg., 22 (2): 91-6, 2006.

17- Park D.D.: Aging Asian upper blepharoplasty and brow. Semin. Plast. Surg., 29 (3): 188-200, 2015.

18- Czyz C.N., Hill R.H. and Foster J.A.: Preoperative evaluation of the brow-lid continuum. Clin. Plast. Surg., 40 (1): 43-5, 2013.

19- Kato H., Mineda K., Eto H., Doi K., Kuno S., Kinoshita K., Kanayama K. and Yoshimura K.: Degeneration, regeneration, and cicatrization after fat grafting: Dynamic total tissue remodeling during the first 3 months. Plast. Reconstr. Surg., 133 (3): 303e-313e, 2014.

20- Suga H., Eto H., Shigeura T., Inoue K., Aoi N., Kato H., et al.: IFATS collection: Fibroblast growth factor-2induced hepatocyte growth factor secretion by adiposederived stromal cells inhibits postinjury fibrogenesis through a c-Jun N-terminal kinase-dependent mechanism. Stem Cells, 27: 238-49, 2009.

21- Mashiko T., Wu S.H., Feng J., Kanayama K., Kinoshita K., Sunaga A., et al.: Mechanical Micronization of Lipoaspirates: Squeeze and Emulsification Techniques. Plast. Reconstr. Surg., 139 (1): 79-90, 2017.

22- Rigotti G., Marchi A., Galiè M., Baroni G., Benati D., Krampera M., et al.: Clinical treatment of radiotherapy tissue damage by lipoaspirate transplant: A healing process mediated by adipose-derived adult stem cells. Plast. Reconstr. Surg., 119 (5): 1409-22, 2007.

23- Sardesai M.G. and Moore C.C.: Quantitative and qualitative dermal change with microfat grafting of facial scars. Otolaryngol. Head Neck Surg., 137: 868-72, 2007.

24- Akita S., Yoshimoto H., Ohtsuru A., Hirano A., Yamashita S.: Autologous adipose-derived regenerative cells are effective for chronic intractable radiation injuries. Radiat. Prot. Dosimetry, 151: 656-60, 2012.

25- Mahdavian Delavary B., van der Veer W.M., van Egmond M., Niessen F.B. and Beelen R.H.: Macrophages in skin injury and repair. Immunobiology, 216 (7): 753-62, 2011.

26- Yoshimura K., Sato K., Aoi N., Kurita M., Hirohi T. and Harii K.: Cell-assisted lipotransfer for cosmetic breast augmentation: Supportive use of adipose-derived stem/stromal cells. Aesthetic. Plast. Surg., 32: 48-55, 2008.

27- Kølle S.F., Fischer-Nielsen A., Mathiasen A.B., Elberg J.J., Oliveri R.S., Glovinski P.V., et al.: Enrichment of autologous fat grafts with ex-vivo expanded adipose tissuederived stem cells for graft survival: A randomised placebocontrolled trial. Lancet., 382 (9898): 1113-20, 2013.

28- Kuno S. and Yoshimura K.: Condensation of tissue and stem cells for fat grafting. Clin. Plast. Surg., 42: 191-7, 2015.

29- Tonnard P.L. and Verpaele A.M.: Discussion: Mechanical Micronization of Lipoaspirates: Squeeze and Emulsification Techniques. Plast. Reconstr. Surg., 139 (1): 91-2, 2017. 